ordnete und 199 Stadtratsabgeordnete in Deutschland. In Schweden verzeichneten sie drei Jahre nach ihrer Entstehung 2011 bereits 215.000 Stimmen.

Trotz ihres Wachstums in rund 40 Ländern folgt ihre Reichweite einer instabilen Logik, die weit entfernt von den gewöhnlichen öffentlichen Auseinandersetzungen anderer Parteien ist. »Wir sind nicht hier, um an die Macht zu kommen«, behauptet Ásta Guthrún Helgadóttir, Piraten-Abgeordnete im isländischen Parlament, »sondern um die Macht zu verteilen« (Witte 2016).

Rodrigo Saturnino ist der brasilianische Parteiführer der Piraten und beobachtet das Phänomen aus wissenschaftlicher Perspektive. Er hat festgestellt, dass es keine »Piratenidentität « gibt, sondern eine »Piratenperspektive als Lebensentscheidung«. Vielmehr als um die Verteidigung spezifischer Ursachen gehe es um »eine Strategie des Widerstands« und man müsse »eine exotische Existenz im Feld der Parteienpolitik« bewahren (Saturnino 2016, 228-229). Seine »kontrapropagandistische« und ironische Ästhetik des Publizierens versucht sicherzustellen, dass die Demokratie »ein weniger undurchsichtiges und besser durchführbares Projekt« wird (Ibid., 233).

\title{
Staatsbürgerschaft neu definiert
}

Was gewinnen wir an den Schwankungen zwischen Erfolgen der zivilen Bewegungen, an der Zersetzung der Parteien und der Verherrlichung ihrer symbolischen Ziele? Was kommt nach den Veränderungen der politischen Kultur und der Formen ihrer Ausübung? Das Colectivo Situaciones schaffte es 2009 nach einer Auswertung der acht Jahre zurückliegenden massiven Aufstände vom Dezember 2001, die von Fernando de la Rúa geführte Regierung bis hin zu deren Auflösung unter Druck zu setzen und den Protest so weit zu intensivieren, bis ihrem Motto getreu »Haut Alle $\mathrm{ab}$ « waren (»Que se vayan todos! «). Das Kollektiv sagte aus, die massive Reaktion auf ökonomische Verdrängung sei »ausgehend von Fragmenten« zustande gekommen. Aus dieser Perspektive bedeutete seine kontrainformative Arbeit laut eigener Aussage die Entdeckung »einer einstimmigen und unbestimmten Willenskraft«. Dabei stell sich allerdings die Frage, wohin dieser unbestimmte Wille führen soll.

Wir beobachten heterogene Widerstände inmitten eines geschwächten und wenig glaubwürdigen Parteiensystems und materialistischen und symbolischen Wirtschaftsmodellen, die uns dort in Cluster einteilen, wo Proteste zerrinnen. Oft täuschen sie eine Teilnahme an der Debatte nur vor: Der Ausblick ist nicht besonders ermutigend, wenn wir ihn ausgehend von der 
Konzeption der modernen oder liberalen Staatsbürgerschaft betrachten. Sie strebt anhand der Gruppierung freier Individuen danach, den Staat und die internationalen Organisationen so zu modifizieren, dass sie dem Gemeinwohl dienen. Der Fortschritt in der Demokratiefrage ist unmöglich, solange wir an von der Vorstellung einer einheitlichen, homogenen Vertretung der sozialen Organisation und der Bürgerschaft festhalten. Schon Michel Foucault lehnte angesichts der Erkenntnis der dezentralisierten Machtlogik und der Fragmentierung von Autorität die Konzeption von Macht als vollkommen integrierte und lokalisierte Struktur ab. Was die Macht so unverständlich macht, so erklärte er in den 1970er-Jahren, ist die Tatsache, dass sie kein einzelner fixierter Punkt sei, kein bestimmter Fokus der Souveränität, von dem abgeleitete und nachgeordnete Punkte ausstrahlen. Anstatt sich die Macht als Institution oder Pyramidenstruktur vorzustellen ist es entscheidend $\mathrm{zu}$ verstehen, dass sie eine strategische Situation darstellt, in der mehrere Kräfte miteinander konkurrieren.

Wenn Foucaults Perspektive sich bereits damals als fruchtbar erwies, so ist ihre Relevanz umso größer bei der Ermittlung, welche Bürgeraktionen sich in der Ära des kognitiven Kapitalismus bewähren. Ich würde sogar behaupten, dass das Zeitalter der algorithmischen Gouvernementalität die in der foucaultschen Konzeption vorgeschlagenen Verschiebungen bis aufs äußerste verstärkt, gewissermaßen von der Macht der Konfrontation zwischen Klassen und Gegnern hin zur (ungleichen) Verteilung von Geräten und Zugangsund Nutzungsformen. Es geht um die Macht als etwas, das man im Sinne einer konzentrierten Substanz über Subjekte oder privilegierte Organismen besitzt. Sie verändert sich in quasi ständig erneuerbare Beziehungen zwischen Subjekten, Artikulationsweisen und der Möglichkeit der Verbindung und Ausschaltung. So sehr Facebook und Amazon das Wachstum im Kommunikationssektor belegen, so sind doch diese Firmen (und noch weniger Herr Zuckerberg) nicht die einzigen Verantwortlichen bei der Informationsgewinnung zur Kontrolle. Verantwortlich sind vielmehr die Strukturen des Datenaustauschs und der Datenverwendung, an denen wir uns als Teil von Milliarden von Nutzern freiwillig beteiligen: Diese Dynamik macht uns zu Dienern der Server. Der Schlüssel dieser Wende ist, dass sie die Rolle des Staates verändert, die im Mittelpunkt Foucaults biotechnologischer Kritik steht. Inzwischen ist der Staat Teil der Zerstreuung der algorithmischen Gouvernementalität.

Die jüngste Geschichte zeigt ein weiteres Zusammenspiel von Akteuren und Kommunikationsmitteln, vermehrte Interaktionen zwischen 
Widerständlern, Aufständischen, Parteien, Staaten, Unternehmen und Algorithmen (den letzteren geben wir hierbei eine Rolle im Sinne von Akteuren oder gar Aktanten nach der Bezeichnung von Bruno Latour). Die foucaultsche Vorstellung, die eine einzige Zusammenführung der heterogenen Widerstände nicht vorsieht, bleibt also gültig. Natürlich erkennen wir eine starke Einschränkung, wenn wir die Ausbreitung und kurze Dauer der Bewegungen mit der wachsenden und zentralisierten Vormachtstellung der transnationalen Unternehmen vergleichen. Wenn wir jedoch die Gouvernementalität als Dispositive verstehen, über die sich die Regierung des Selbst und die Regierung der anderen auszudrücken vermögen, dann ist es möglich, die Beziehungen zwischen den Bürgern und die zwischen den Bürgern-Nutzern mit den Unternehmen in der Gesamtheit der Institutionen, Vorgänge, Berechnungen und Taktiken, die die Machtausübung im Zeitalter der Algorithmen neu formulieren, zu denken.

Aus der modernen, liberalen Perspektive befindet sich die aktuelle Staatsbürgerschaft in einem gebrochenen Zustand. Wir üben sie aus, indem wir beispielsweise unsere besonderen Rechte als Indigene, Frauen, Jugendliche und Nachbarn verteidigen. Wir sind aber zu schlecht vernetzt und haben eine geringe Kapazität zur dauerhaften Verbündung. Währenddessen haben die Staaten ihre Macht missbraucht, und das Stimmrecht - der letzte Akt, der uns als Bürger vereint und uns näher an die Gleichberechtigung bringt - ist zu einer nahezu leeren Idee verkommen. Die soziodigitalen Netzwerke, die uns verbinden, sind virtuell. Und dies im doppelten Sinne des Wortes: Sie verdoppeln die Materialität des »Realen« und sie kommen über mögliche Handlungen nicht hinaus. Die Umwandlung von Bewegungen in Parteien oder ihre Integration in Volksfronten kann helfen, aber sie löst die Gleichsetzung zwischen Konzentration und Zerstreuung nicht auf.

Genauso wie Unternehmen die Verbraucher nicht mehr standardisieren und sich fragen, was mit den Anderen zu tun ist, müssen wir als Bürger*innen herausfinden, was wir als »die Anderen« bewirken können. Eine Akzeptanz der Unsicherheit der Unterschiede löst die Probleme des Ganzen nicht. Die Unternehmen nutzen Unterschiede für ihre Strategien, um Unterscheidungen zu generieren. Aber ihre Designstile, Werbung und Warenzirkulation fließen in der Buchhaltung zusammen, die in ihrer Verwaltung integriert ist. Als Verbraucher und Nutzer können wir in unserer Bürgerrolle unsere Rechte als Minderheiten verteidigen. Genau wie Indigene, Frauen, Homosexuelle und Bewohner aus marginalisierten Stadtteilen stellen wir in der heutigen Welt eine Minderheit dar. Wenn wir dabei noch erkennen, dass wir in unglei- 
chen Unterschieden leben, können unsere Aktionen umso konsistenter und stärker werden (Fernández 2008). Wie Ana María Fernández, erklärt, entsteht die Selbstermächtigung aus einer simultanen Anerkennung von Unterschieden, die hierarchisch über ungleichmachende Biologismen, Ökonomismen und Technokratismen integriert werden. Der Begriff der Staatsbürgerschaft kann so angewandt zur Überwindung ethnischer, geschlechterspezifischer oder nationalitätsspezifischer Essenzialismen angewandt werden, die in der Regel mit getrennten Identitätskonstruktionen zusammenhängen. Er kann der Entwicklung einer gemeinsamen oder konvergierenden Emanzipationsstrategie dienen.

Noch werden wir nicht vollkommen durch Algorithmen ersetzt. Aber die Möglichkeit, uns als Subjekte im Bündnis mit anderen zu erholen wird durch die Parteien gehemmt, die keine Verbindung mehr zu ihrer Klientel haben. Auch die Medien, die uns von Entscheidungsplattformen weggelockt haben, tragen hierzu bei. Gleiches gilt für die politischen Entscheidungen, die gegenwärtig der Macht anonymer Entitäten nachgeben. Es sollte also herausgefunden werden, ob in den Bewegungen von heute noch ein Fünkchen politisches Leben enthalten ist, das nicht nur auf Daten basiert, wie es in den materiellen und symbolischen Unternehmenswirtschaften der Fall ist. Denn dann könnte das wahrhaftige Sich-Austauschen zu einem neuen, sinnhaften Abenteuer führen, einem Abenteuer des Sinns. 
